\title{
Sink Mobility Oriented Data Aggregation using ETSSE Protocol in Heterogeneous Wireless Sensor Network Having Multiple Cluster Association
}

\author{
Sunil Kumar Bisaiya \\ M.Tech Student \\ Dept. of Computer Science \\ and Engineering \\ Delhi Technological University, \\ Delhi (India)
}

\author{
S. K. Saxena, PhD \\ Senior Faculty \\ Dept. of Computer Science and \\ Engineering \\ Delhi Technological University, \\ Delhi (India)
}

\begin{abstract}
In this article, an approach for heterogeneous environment of three types of nodes with different energy levels namely advance nodes, intermediate nodes and normal nodes is proposed. Energy efficiency of the proposed approach can be improved through Multiple Cluster Heads. The mobile sink node and mobile sensor nodes can directly reach cluster heads and collect data from it. Mobile sink reduces the energy consumption arises due to routing of data to the static sink, Cluster Head or Base Station. Path of sink node is optimized to reach the cluster heads. Mobility of sink via shortest path reduces delay of data delivery. Proposed approach with sink mobility and without sink mobility are compared by conducting simulation in NS2. Performance of this approach is evaluated with appropriate metrics.
\end{abstract}

\section{General Terms}

Wireless Sensor Networks, Sensor node, Heterogeneous environment, Base Station(BS)

\section{Keywords}

Clustering, Mobile Sink (MS), Cluster Head, Stable Election Protocol(SEP),Energy-efficiency, Network Lifetime, Wireless Sensor Network(WSN), Enhanced Threshold Sensitive Stable Election Protocol(ETSSEP), Rendezvous Points(RPs)

\section{INTRODUCTION}

Wireless Sensor Networks

A wireless sensor network is a collection of nodes organized into a cooperative network consisting of tiny battery powered sensor nodes to monitor physical or environmental conditions, like sound, vibration, pressure, temperature, motion or pollutants at different locations[1].Every node has its own processing capability, may contain multiple types of memory (program, data and flash memories), have a RF transceiver (usually with a single omni-directional antenna), have a power source (e.g., batteries and solar cells), that mainly accommodates numerous sensors and actuators. Such systems can revolutionize the way to live and work.

Currently, wireless sensor networks are beginning to be deployed at an accelerated pace just like the Internet. This new skill is stimulating with unrestrained prospective for numerous application areas. In this network, node senses the data from impossibly accessible area and sends their report to the base station also called the sink.

Energy is a paramount concern to wireless sensor networks (WSNs) that must operate for an extended period of time on limited power supplies such as batteries. A major portion of energy expenditure of WSNs is attributed to multi-hop wireless communications. For instance, a mobile base station
(BS) may roam about a sensing field and collect data from sensor nodes through short-range communications.

The major performance bottleneck of WSNs with a mobile BS is the increased latency in data collection. The typical speed of practical mobile sensor systems about $0.1-2 \mathrm{~m} / \mathrm{s}$. As a result, it takes a mobile BS hours to tour a large sensing field, which cannot meet the delay requirements of many sensing applications. The low movement speed is a fundamental design constraint for mobile BSs because increasing the speed will lead to significantly higher manufacturing cost and power consumption.

MS (Mobile Sink): Mobile Sink [18] traverse through the entire WSN to collect data from the sensor nodes. A mobile sink move across a sensing field. It can either use the data autonomously or can send it to remote stations or to users via wireless communication. The route between source and MS is through multi-hop but the path is dynamic since the MS keeps changing its position. The usage of mobile sink in the wireless sensor networks reduces the energy consumption of the nodes and to prevent the formation of energy holes in wireless sensor networks. Sink node collects data from the sensor nodes as shown in fig.1.

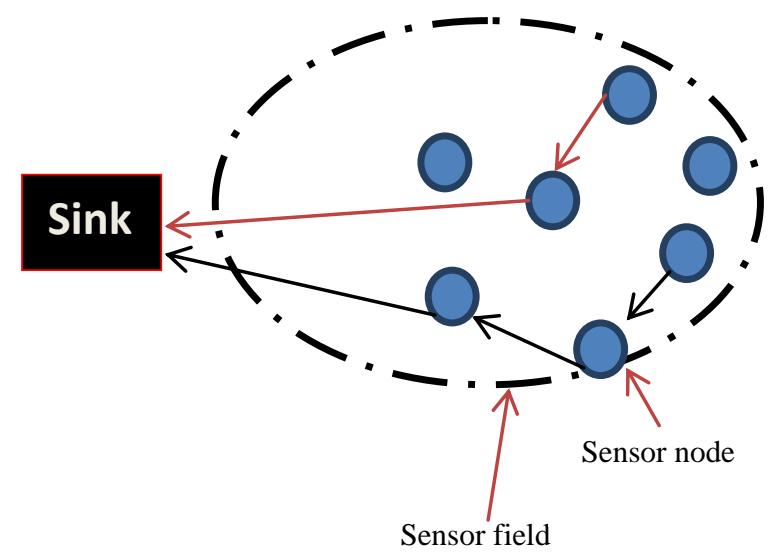

Figure 1: Multi-hop transmission

\section{LITERATURE REVIEW}

In WSN, Sensor nodes sense the data from impossibly accessible area, cooperatively forward the sensed data to the sink or base station via multi-hop wireless communication and sends their report to the base station also called the sink[20]. The nodes in wireless sensor networks can be mobile or stationary and deployed in the area through a proper or random deployment mechanism. Different types of attributes of the sensor nodes are energy consumption, size, lifetime of 
operation, power level etc. WSN is widely used in various domains like medical diagnoses, industrial processes, military surveillances and traffic management. Over the few last decades, energy conservation has been a major objective for WSN. Life of a wireless sensor depends on its battery lifetime. According to radio energy dissipation model illustrated in Fig. 2 [7], the energy expanded by transmitting $\mathrm{K}$ bit message over a distance $\mathrm{d}$ is given by:

$$
E_{T x}(K, d)= \begin{cases}K \cdot E_{\text {elec }}+K \cdot E_{f s} * d^{2} & \text { if } d<d_{0} \\ K \cdot E_{\text {elec }}+K \cdot E_{\text {amp }} * d^{4} & \text { if } d \geq d_{0}\end{cases}
$$

Where $E_{\text {elec }}$ is the energy dissipated per bit to run the transmitter or the receiver and $\mathrm{d} 0$ denotes the threshold distance and calculated as:

$$
d_{0}=\sqrt{\frac{E_{f s}}{E_{a m p}}}
$$

There are two different radio models which are used: the free space model $\left(\mathrm{E}_{\mathrm{fs}}\right)$ and the multipath fading channel model $\left(\mathrm{E}_{\mathrm{amp}}\right)$. The distance between the transmitter and receiver is $\mathrm{d}$. If $\mathrm{d}$ is less than $\mathrm{d}_{0}$, free space model is used; otherwise multipath fading channel is used. $\mathrm{E}_{\mathrm{Rx}}$ is the energy dissipated for receiving $\mathrm{K}$ bits and calculated as:

$$
E_{R x}(K)=K . E_{\text {elec }}
$$

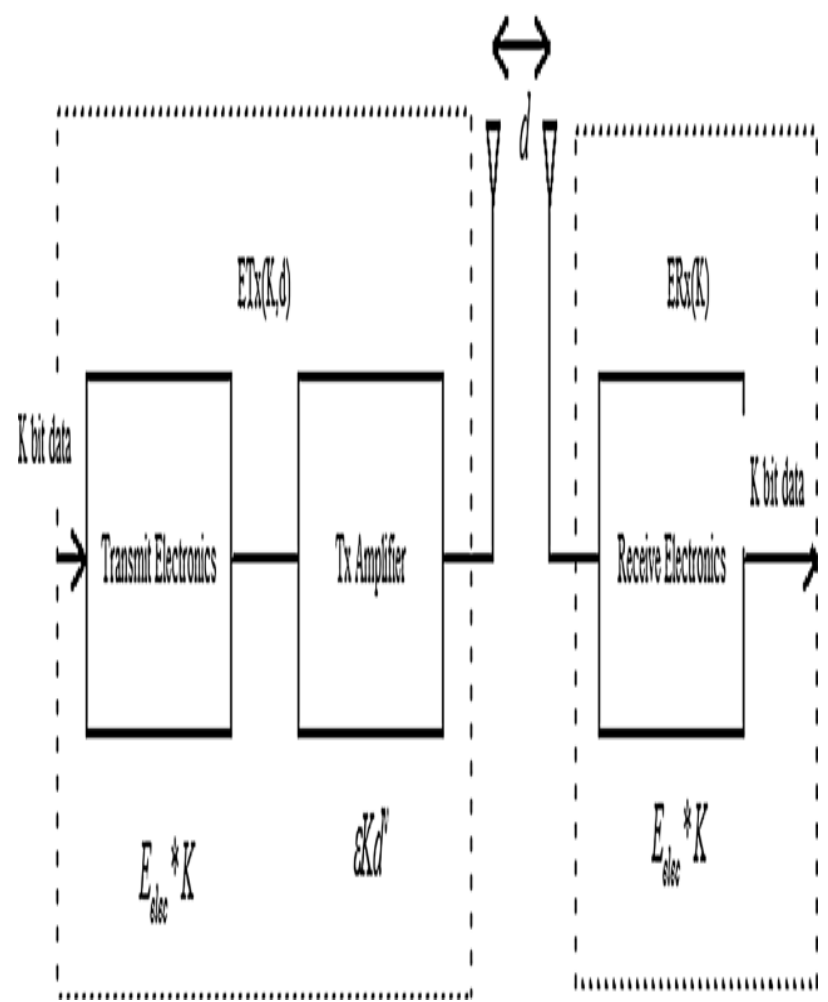

Figure 2: Radio energy dissipation model

Wireless sensor networks consists battery operated sensor nodes. Due to this fact, there is a lot of focus on finding the best energy efficient algorithms for these types of networks. One of the best known approaches to minimize the energy consumption is clustering as shown in fig.3.Clustering is mainly divided into three phases: cluster head $(\mathrm{CH})$ selection, cluster formation and data transmission. The first part is $\mathrm{CH}$ selection, in which cluster heads are elected on the basis of the probability of being a cluster head [3]. Once the cluster head is elected, it broadcasts advertisement to the nodes to form a cluster. After

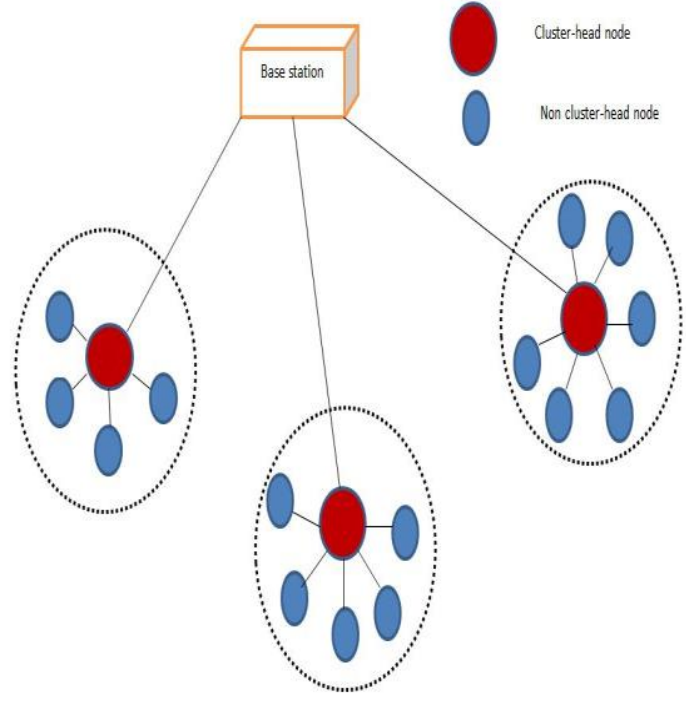

Figure 3: Architecture of cluster based network

cluster formation, the sensor nodes in the cluster send their sensed value to the cluster head during their time slots. The cluster head receives all the data from sensor nodes and aggregate it before transmitting to the sink. Clustered sensor networks can be classified into two categories in terms of energy. In a heterogeneous network, different nodes are at the different energy levels while in a homogeneous network, all the nodes are having the same energy levels

Clustering drastically reduces the energy consumption and improves the network lifetime. In this approach different protocols are used. The protocols for such types of networks must be energy efficient due to non-replacement of batteries in nodes after its deployment. Protocols are classified into two categories according to their applications, proactive protocols and reactive protocols. In former, sensor nodes sense the data from different locations and continuously transmit that data to the cluster head, then cluster head transmits to the base station either it is needed or not, while in later, the cluster head transmits the data only if there is a drastic change in the sensed value.

In routing protocols, cluster head election reduces energy consumption and enhances the network life time. Classical approach like direct transmission (DT) and minimum energy transmission (MTE) does not guarantee well distribution of energy load of sensor nodes. In DT approach, sensor nodes directly transmit the data to the base station, so the nodes far away from sink will die first, while in case of MTE, data is routed over minimum cost routes, where this cost reflects the transmission power. In the MTE, nodes near the base station act as relays with higher probability than nodes that are far from the base station. So the nodes near the sink will die first. Under both the cases, a part of the area will not be monitored. A solution for this problem, called LEACH, guarantees that the energy load will be well distributed by creating clusters dynamically. LEACH is one of the most famous and oldest protocols based on clustering mechanism. In LEACH, cluster heads are dynamically elected according to the election probability. Each node becomes a cluster head, once every one epoch of rounds, by rotating the $\mathrm{CH}$ role uniformly among all nodes. It is a homogeneous wireless sensor network protocol. LEACH is not as successful for heterogeneous networks. So for heterogeneous networks, many different protocols are considered, Stable Election Protocol (SEP) is one of them. SEP [16] is a reactive routing protocol for heterogeneous 
networks. It is cluster based routing protocol, in which cluster head is elected randomly according to the election probability. It performs well for heterogeneous networks. The Proper utilization of energy affects the network performance.

A sink, which may be static/mobile, acts like a connection between users and the network. One can get the required information from the network by sending the queries and collecting results from the sink[20]. The sensor nodes can also communicate among themselves using radio signals.

Also each sensor node in a wireless sensor network (WSN) is resource constrained. i.e. they have limited processing speed, battery. In many applications replacing sensor node's batteries is difficult or impractical. Therefore it is very important to efficiently utilize sensor node's energy to accomplish a specific mission.

Mobile-Sink Tour : Travelling Salesman problem (TSP) used to find a shortest path for visiting all Cluster Heads by a Mobile-Sink node. A mobile sink that preferentially visits areas of RP will prevent energy holes from forming in a WSN. In clustering purpose only, all the sensor nodes send its data's to cluster head and cluster head sends the data to mobile sink node that travel along the network to collect the data [19]. This process to effectively save the energy of network.

\section{RELATED WORK}

Heinzelman [7] proposed Low Energy Adaptive clustering Hierarchy (LEACH)protocol. LEACH is the first hierarchical, reactive routing protocol for WSN. It is one of the most popular routing protocols for WSN. LEACH is a protocol, which is implemented for homogeneous networks (all sensor nodes are equipped with same amount of energy).The LEACH protocol elects cluster heads; forms cluster from the sensor nodes, aggregate the data and transfers the aggregated data to the base station. This lowers the energy consumption since only the cluster head is transmitting the data to the base station rather than all the sensor nodes in the network. In LEACH, the assumption is that, the base station or the sink is fixed. The basic idea of this protocol is to organize the nodes into clusters[14], and it selects a cluster head for each cluster randomly and thus consumes energy uniformly. A node elects itself as a cluster head randomly and it is done in a way that each node becomes a cluster head once in an epoch. This decision is made by the node itself, in the beginning every node choose a random number between 0 and 1, and computes a threshold $\mathrm{T}(\mathrm{s})$. A node becomes a $\mathrm{CH}$ for the current round if the randomly chosen number is less than the threshold as shown in eq.(4):

$$
\mathrm{T}(\mathrm{n})=\mathrm{p} /(1-\mathrm{p} *(\mathrm{r} \bmod )(1 / \mathrm{p})) ; \text { if } \mathrm{n}
$$

$$
0 \text {; otherwise (4) }
$$

Where, $\mathrm{p}$ is the cluster head probability, $\mathrm{r}$ is the current round number and $G$ is the group of nodes that have not been cluster- heads in the last $1 / \mathrm{p}$ rounds. This mechanism improves the energy consumption because the transmission is done by only such cluster heads rather than the direct transmission of all the sensor nodes. Optimal number of cluster heads is estimated to be $5 \%$ of the total number of nodes. For homogeneous networks, LEACH performs very well, but for heterogeneous networks it is not suitable. So, to overcome this weakness Smaragdakis proposed Stable Election Protocol (SEP) for heterogeneous wireless sensor network which also guarantees network's reliability. SEP extends LEACH in assigning election probabilities for nodes to become $\mathrm{CHs}$ according to the initial energy of a node. SEP is based on weighted election probability. Each normal node becomes a cluster head once every $1 / \mathrm{P}_{\text {opt. }}\left(1+\alpha . \mathrm{m}_{\text {) }}\right.$ rounds per epoch. Each advance node becomes a cluster head $(1+\alpha)$ every $1 / \mathrm{P}_{\text {opt. }(1+\alpha . \mathrm{m}) \text { rounds per epoch. }}$

In this protocol, two types of nodes are considered advance nodes and normal nodes. We consider that a fraction $(\mathrm{m}$ advance nodes) of total number of $\mathrm{n}$ nodes is provided with an additional energy factor $\alpha$, between the advance node and normal node. Suppose $\mathrm{E}_{0}$ is the initial energy of normal node than the energy of advance node will be $\mathrm{E}_{0}(1+\alpha)$ So, the total energy of the system will be increased by a factor of $\left(1+\alpha . m_{)}\right.$ Now, the threshold for normal node $\mathrm{T}\left(\mathrm{s}_{\mathrm{nrm}}\right)$, and for advance node $\mathrm{T}\left(\mathrm{s}_{\text {adv }}\right)$ will be:

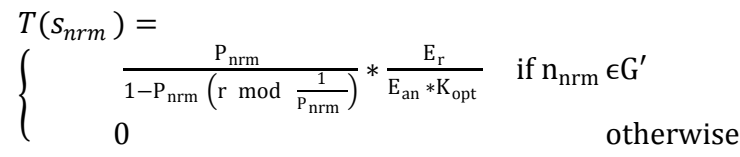

$T\left(s_{a d v}\right)=$

$\left\{\begin{array}{r}\frac{\mathrm{P}_{\text {adv }}}{1-\mathrm{P}_{\text {adv }}\left(\mathrm{r} \text { mod } \frac{1}{\mathrm{P}_{\text {adv }}}\right)} * \frac{\mathrm{E}_{\mathrm{r}}}{\mathrm{E}_{\text {aa }} * \mathrm{~K}_{\text {opt }}} \text { if } \mathrm{n}_{\text {adv }} \in \mathrm{G}^{\prime \prime \prime} \\ 0 \quad \text { otherwise }\end{array}\right.$

where, $p_{n r m}$ and $p_{a d v}$ are the weighted probabilities for normal and advance nodes, $r$ is current round, $G^{\prime}$ is the set of normal nodes that have not become cluster head within the last $1 / \mathrm{P}_{\mathrm{nrm}}$ round of epoch and G" is the set of advance node that have not become cluster headwithin the last $1 / \mathrm{P}_{\mathrm{adv}}$ round of epoch.

So, SEP increases the stability period and network life time due to the presence of advance nodes. For increasing the better stability and network life time an extension of SEP is proposed [8], in which three levels of heterogeneity; normal nodes, intermediate nodes and advance nodes are considered. Where, advance nodes have energy greater than all the other nodes and a fraction of nodes which have more energy than the normal nodes and less energy than advance nodes, called the intermediate nodes, while rest of the nodes are called the normal nodes. In this approach, cluster heads are also selected depending on the election probability of each type of node as in SEP. Afterwards, several reactive routing protocols were proposed for heterogeneous networks following the goal of SEP. A reactive routing protocol is used for time critical applications, such as Distributed Energy Efficient Clustering (DEEC), Threshold Sensitive Energy Efficient Routing (TEEN), and Heterogeneity-aware Hierarchical Stable Election Protocol (HSEP). In TEEN,] transmission is done only if a severe change occurs in the value. It is a threshold sensitive protocol based on two thresholds, hard threshold and soft threshold. When, the sensed value becomes equal or greater than the hard threshold, nodes turn on their transmitters and transmit the values to the $\mathrm{CHs}$. And, at the second time they transmit only if the difference between current sensed value and previously saved value is equal or greater than the soft threshold. So, the hard threshold and soft threshold tries to reduce the transmission by allowing the nodes to transmit sensed attributes within a range. So, the energy consumption is reduced, the lifetime and the stability of the system is improved. The main drawback of this scheme is that the nodes will never communicate if the thresholds are not reached, hence the user will not get any data from the network and unable to know even if all the nodes die. As described in , a new approach proposed is Threshold sensitive Stable Election Protocol, which is also a reactive protocol with three level of heterogeneity. In TSEP, CHs selection is threshold based. In this protocol three types of nodes with 
different energy levels are considered advance nodes, intermediate nodes and normal nodes. So, TSEP increased stability and network lifespan due to three levels of heterogeneity and reactive routing, it also increases the network throughput.

\section{PROPOSED WORK}

ETSSEP is a cluster based reactive routing protocol with three level of heterogeneity. For three levels of heterogeneity, nodes with different energy levels are: advance nodes, intermediate nodes and normal nodes. The energy of advance nodes are greater than all other nodes and a fraction of nodes which have more energy than normal node and less energy than advance nodes are called intermediate nodes, while rest of the nodes are called normal nodes. Intermediate nodes have $\beta$ times more energy than normal nodes, advance nodes have $\alpha$ times more energy than normal nodes and we assume that $\beta=\alpha / 2$. In ETSSEP the total energy distributed over different types of nodes is computed as:

The Cluster head is elected by checking the probability of each node. The probabilities of different types of nodes for electing a Cluster head in case of three level of heterogeneity. The proportion of advance nodes to the total number of nodes $\mathrm{n}$, and $\mathrm{b}$ is the proportion of intermediate nodes of the total no of nodes n. Initially, each node can become a Cluster head with a certain probability. Nodes that are elected to be cluster head in the current round cannot be Cluster head in the same epoch. When Cluster head selection is done another parameter threshold is taken into consideration. Each node generates a random number between 0 and 1 , if generated value is less than the threshold than the node will become Cluster head. In the proposed approach, have adjusted the value of threshold for selection of the Cluster head, based on the ratio of residual energy of the node and average energy of the network and optimal number of clusters per round. So, only the node with the highest energy will become the Cluster head. The efficient routing protocol in a cluster plays an important role in energy saving and stability of the cluster and its nodes. The existing system is threshold sensitive stable election (TSEP) protocol which does not consider the energy levels of the nodes for the cluster head $(\mathrm{CH})$ selection, during threshold calculation and $\mathrm{CH}$ is still probability based in TSEP protocol.

In this paper, a mobile sink based data collection approach is proposed that explores the controlled mobility of the sensor nodes[23]. Mobile Sink will aggregate data from Cluster heads via single or multi hop communication. Cluster heads collect data from the originating sensor nodes. This approach has several key advantages. First, a broad range of desirable trade-offs between energy consumption and communication delay can be achieved by jointly optimizing the choices of RPs, motion path of BS and data transmission routes. Second, the use of RPs enables the BS to collect a large volume of data at a time without traveling a long distance, which mitigates the negative impact of slow speed of BS on overall network throughput. Third, mobile nodes communicate with the rest of the network through RPs at scheduled times, which minimizes the disruption to the network topology caused by mobility. Energy of a node can be optimized in WSN by taking the advantage of mobile sink or mobile agents. Many approaches using mobile sink have demonstrated that energy usage can be optimized significantly in the phenomenon area from where the sink would collect the sensed readings from the sensor nodes via single or multi hop communication.

\section{Advantages}

It dynamically changes cluster head election probability .It improves the throughput and network lifetime of the network.

\subsection{Multiple Cluster Association}

A node receives Cluster Head advertisement message from multiple cluster head. It joins to the one which is closer to itself and send join request message to the corresponding cluster head. The nodes that are neighbors of multiple clusters, divides the data and send it to multiple clusters heads based on dependency degree. While multiple cluster association maintains the balanced energy consumption, network lifetime is improved.

Dependency Degree $=($ Total distance-Distance to each cluster head)/Total distance all neighbor cluster head.

\subsection{Mobile Sink Data Gathering}

Using mobile sinks, sensors could communicate with a sink when it gets closer, thus using shorter hop-by-hop data delivery paths. Mobile sinks can also change their location when the nearby sensors' energy becomes low. In this way, the set of sensors located near sinks change over time, the energy consumption is balanced, and the network lifetime is prolonged.

A sink node has more resources in terms of power, computation and mobility[21]. A cluster head manages the sensors in its cluster, gathers information from them, and forwards data to/from the sink.

The main objective of these algorithms is to design mechanisms that prolong network lifetime by employing mobile sinks to gather information from the sensors.

Assume that $\beta=\alpha / 2$. In ETSSEP the total energy distributed over different types of nodes is computed as:

For Normal Node

$$
\mathrm{E}_{\mathrm{nrm}}=\text { n.b. }(1+\beta)
$$

For Intermediate Node

$$
\mathrm{E}_{\mathrm{int}}=\quad \mathrm{n} \cdot(1-\mathrm{m}-\mathrm{b} \cdot \mathrm{n}) \cdot \mathrm{E}_{0}
$$

For Advance node

$$
\mathrm{E}_{\mathrm{adv}}=\mathrm{n} \cdot \mathrm{m}(1+\alpha) \cdot \mathrm{E}_{0}
$$

Total energy $\mathrm{E}_{\text {total }}$ for all the nodes is calculated as

$$
\begin{aligned}
\mathrm{E}_{\text {total }} & =\mathrm{n} \cdot(1-\mathrm{m}-\mathrm{b} \cdot \mathrm{n}) \cdot \mathrm{E}_{0}+\mathrm{n} \cdot \mathrm{m}(1+\alpha) \cdot \mathrm{E}_{0}+\mathrm{n} \cdot \mathrm{b} \cdot(1+\beta) \\
& =\mathrm{n} \cdot \mathrm{E}_{0}(1+\mathrm{m} \cdot \alpha+\mathrm{b} \cdot \beta)
\end{aligned}
$$

Where, $\mathrm{m}$ and $\mathrm{b}$ denotes the advance nodes and intermediate nodes fraction of total number of nodes $n$.

\section{MODULES DESCRIPTION \\ 5.1 Measurement of Total Energy Using ETSSEP Protocol}

ETSSEP is a cluster based reactive routing protocol with three level of heterogeneity. For three levels of heterogeneity, nodes with different energy levels are: advance nodes, intermediate nodes and normal nodes. The energy of advance nodes are greater than all other nodes and a fraction of nodes which have more energy than normal node and less energy than advance nodes are called intermediate nodes, while rest of the nodes are called normal nodes. 
Intermediate nodes have $\beta$ times more energy than normal nodes, advance nodes have $\alpha$ times more energy than normal nodes.

\subsection{Cluster Head Selection of ETSSEP Protocol}

In ETSSEP, the calculated probability depends on the residual energy of node and average energy of the network at round $r$. The average energy of network at round $\mathrm{r}$ is estimated as:

$$
E(r)=\frac{1}{N} E_{\text {total }}\left(1-\frac{r}{R}\right)
$$

Where,

$r$ is the current round

$\mathrm{N}$ is total number of nodes,

$\mathrm{E}_{\text {total }}$ is total initial energy of the heterogeneous network and

$\mathrm{R}$ denotes the total rounds of the network calculated as:

$R=\frac{E_{\text {total }}}{E_{\text {round }}}$

The energy dissipated in the network in a particular round is denoted as $\mathrm{E}_{\text {round }}$.

The cluster head is elected by checking the probability of each node. The probabilities of different types of nodes for electing a cluster head in case of three level of heterogeneity are:

$$
\begin{aligned}
\mathrm{P}_{\mathrm{nrm}}= & \frac{\mathrm{P}_{\mathrm{opt}}}{1+\mathrm{m} \cdot \alpha+\mathrm{m} \cdot \beta} \\
\mathrm{P}_{\mathrm{int}}= & \frac{\mathrm{P}_{\mathrm{opt}}(1+\beta)}{1+\mathrm{m} \cdot \alpha+\mathrm{m} \cdot \beta} \\
\mathrm{P}_{\mathrm{adv}}= & \frac{\mathrm{P}_{\mathrm{opt}}(1+\alpha)}{1+\mathrm{m} \cdot \alpha+\mathrm{m} \cdot \beta}
\end{aligned}
$$

Where,

$\mathrm{m}$ is the proportion of advance nodes to the total number of nodes $\mathrm{n}$,

$\mathrm{b}$ is the proportion of intermediate nodes of the total no of nodes $\mathrm{n}$.

Initially, each node can become a cluster head with a certain probability. When cluster head selection is done another parameter threshold is taken into consideration. Each node generates a random number between 0 and 1 , if generated value is less than the threshold than the node will become cluster head. In the proposed approach, have adjusted the value of threshold for selection of the cluster head, based on the ratio of residual energy of the node and average energy of the network and optimal number of clusters per round. So, only the node with the highest energy will become the cluster head.

The threshold is set as different types of nodes:

$$
\begin{aligned}
& T\left(s_{\text {nrm }}\right) \\
& =\left\{\begin{array}{cc}
\frac{\mathrm{P}_{\mathrm{nrm}}}{1-\mathrm{P}_{\mathrm{nrm}}\left(\mathrm{r} \bmod \frac{1}{\mathrm{P}_{\mathrm{nrm}}}\right)} * \frac{\mathrm{E}_{\mathrm{r}}}{\mathrm{E}_{\mathrm{an}} * \mathrm{~K}_{\mathrm{opt}}} & \text { if } \mathrm{n}_{\mathrm{nrm}} \in \mathrm{G}^{\prime} \\
0 & \text { otherwise }
\end{array}\right.
\end{aligned}
$$

$$
\begin{aligned}
& T\left(s_{\text {int }}\right) \\
& =\left\{\begin{array}{cc}
\frac{\mathrm{P}_{\text {int }}}{1-\mathrm{P}_{\mathrm{int}}\left(\mathrm{r} \bmod \frac{1}{\mathrm{P}_{\text {int }}}\right)} * \frac{\mathrm{E}_{\mathrm{r}}}{\mathrm{E}_{\mathrm{ai}} * \mathrm{~K}_{\mathrm{opt}}} & \text { if } \mathrm{n}_{\mathrm{int}} \in \mathrm{G}^{\prime \prime} \\
0 & \text { otherwise }
\end{array}\right. \\
& =\left\{\begin{array}{c}
\frac{\mathrm{P}_{\mathrm{adv}}}{1-\mathrm{P}_{\mathrm{adv}}\left(\mathrm{r} \bmod \frac{1}{\mathrm{P}_{\mathrm{adv}}}\right)} * \frac{\mathrm{E}_{\mathrm{r}}}{\mathrm{E}_{\mathrm{aa}} * \mathrm{~K}_{\mathrm{opt}}} \\
0 \quad \text { if } \mathrm{n}_{\mathrm{adv}} \in \mathrm{G}^{\prime \prime \prime}
\end{array}\right.
\end{aligned}
$$

Where,

G', G' and G', are the set of normal, intermediate and advance nodes $\mathrm{T}_{\mathrm{nrm}}, \mathrm{T}_{\mathrm{int}}$ and $\mathrm{T}_{\mathrm{adv}}$ are the threshold applied to the population of normal nodes, intermediate nodes and advance nodes.

Er is the residual energy of any node.

$E_{a n i}, E_{a i}$ and $E_{a a}$ is the average energy of normal node, intermediate node and advance node.

$\mathrm{K}_{\text {opt }}$ is the optimal number of clusters per round. The cluster head is elected, it broadcasts advertisement to the nodes to form a cluster. After cluster formation, the sensor nodes in the cluster send their sensed value to the cluster head during their time slots. The cluster head receives all the data from sensor nodes and aggregate it before transmitting to the base station.

\subsection{Performance Evaluation}

The performance of the proposed approach using ETSSEP in Heterogeneous WSN is computed on following parameters:

\section{Throughput}

It is the amount of time taken by the packet to reach the destination.

Throughput $($ bits/s) $=$ Total Data / Data Transmission duration

\section{Network Lifetime}

Network lifetime is the time at which the first network node runs out of energy to send a packet, because to lose a node could mean that the network could lose some functionalities.

\section{Stability Period}

Stability of the protocol is the time interval from the start of network until the death of first node is called stable region.

\section{Instability Period}

Instable period of protocol is the time from the death of first node until the death of last node also called the unstable region.

\section{SIMULATION RESLUTS AND CONCLUSION}

Scenario: Proposed scheme with sink mobility and existing scheme without sink mobility schemes are compared for the scenarios of varying number of rounds. Scenario is kept same for both protocols with same topology and energy. Totally 4 simulation runs are made by varying number of rounds as 1,2 , 3 , and 4. Parameters such as network lifetime, stability period, instability period and throughput are computed and plotted as $\mathrm{X}$ graph. 
Table 1: Simulation Parameters

\begin{tabular}{|l|l|}
\hline SIMULATOR & $\begin{array}{l}\text { Network } \\
\text { Simulator 2 }\end{array}$ \\
\hline $\begin{array}{l}\text { NUMBER OF } \\
\text { NODES }\end{array}$ & Random \\
\hline TOPOLOGY & Random \\
\hline $\begin{array}{l}\text { INTERFACE } \\
\text { TYPE }\end{array}$ & $\begin{array}{l}\text { Phy/Wireless } \\
\text { Phy }\end{array}$ \\
\hline MAC TYPE & 802.11 \\
\hline QUEUE TYPE & $\begin{array}{l}\text { Drop } \\
\text { tail/Priority } \\
\text { Queue }\end{array}$ \\
\hline $\begin{array}{l}\text { ANTENNA } \\
\text { TYPE }\end{array}$ & Omni Antenna \\
\hline $\begin{array}{l}\text { PROPAGATIO } \\
\text { N TYPE }\end{array}$ & Two ray Ground \\
\hline $\begin{array}{l}\text { ROUTING } \\
\text { PROTOCOL }\end{array}$ & AODV \\
\hline $\begin{array}{l}\text { TRANSPORT } \\
\text { AGENT }\end{array}$ & UDP \\
\hline $\begin{array}{l}\text { APPLICATION } \\
\text { AGENT }\end{array}$ & CBR \\
\hline $\begin{array}{l}\text { INITIAL } \\
\text { ENERGY }\end{array}$ & 1 Joules \\
\hline AREA & $500 *$ 500 \\
\hline TIMULATION & 150 seconds \\
\hline
\end{tabular}

Cluster Head Selection Based on Highest Residual Energy: Each cluster member sends join request message to corresponding cluster head from which it received the signal with high strength.

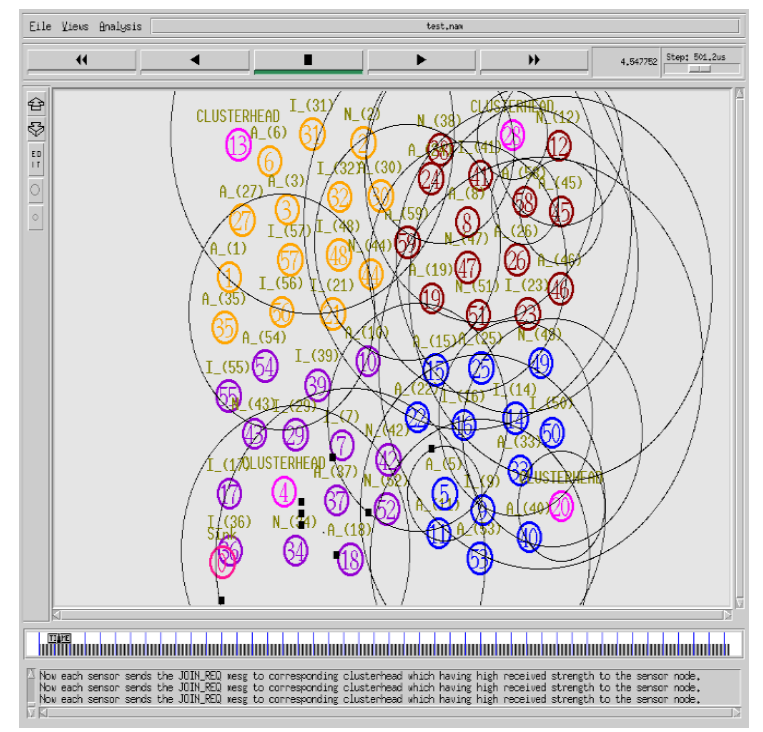

\section{Figure 4:Cluster Head SelectionCluster Head data aggregation for Mobile Sink:}

Cluster member sends the sensed data to their cluster head. Then cluster head aggregates all sensed data and waits to send it to sink.

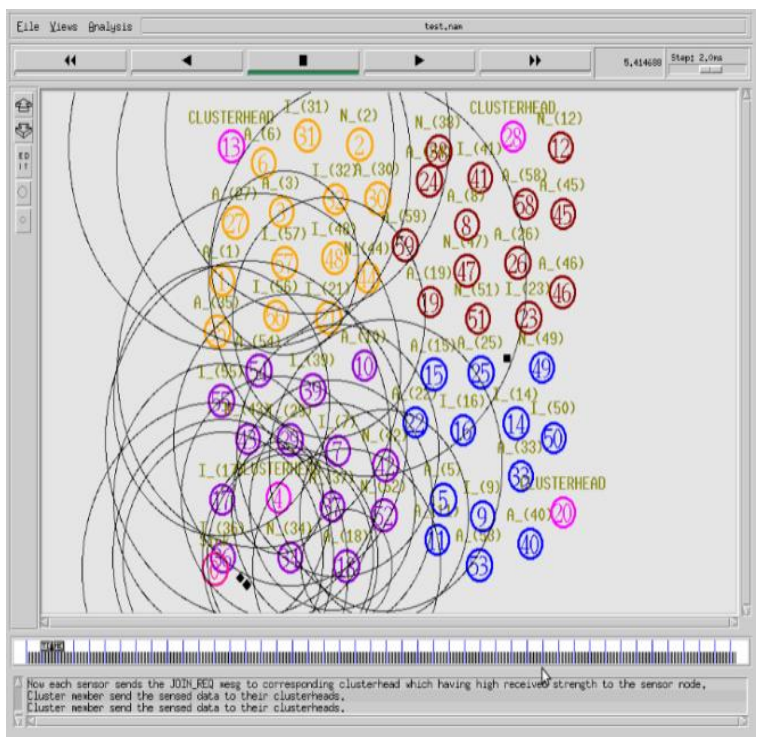

Figure 5: Cluster member sending data to $\mathrm{CH}$

Sink Mobility Sink travel via optimal path and collects data.

Once the mobile sink arrives at the cluster head, it collects data from sensor nodes via the cluster head.

\section{Without Sink Mobility}

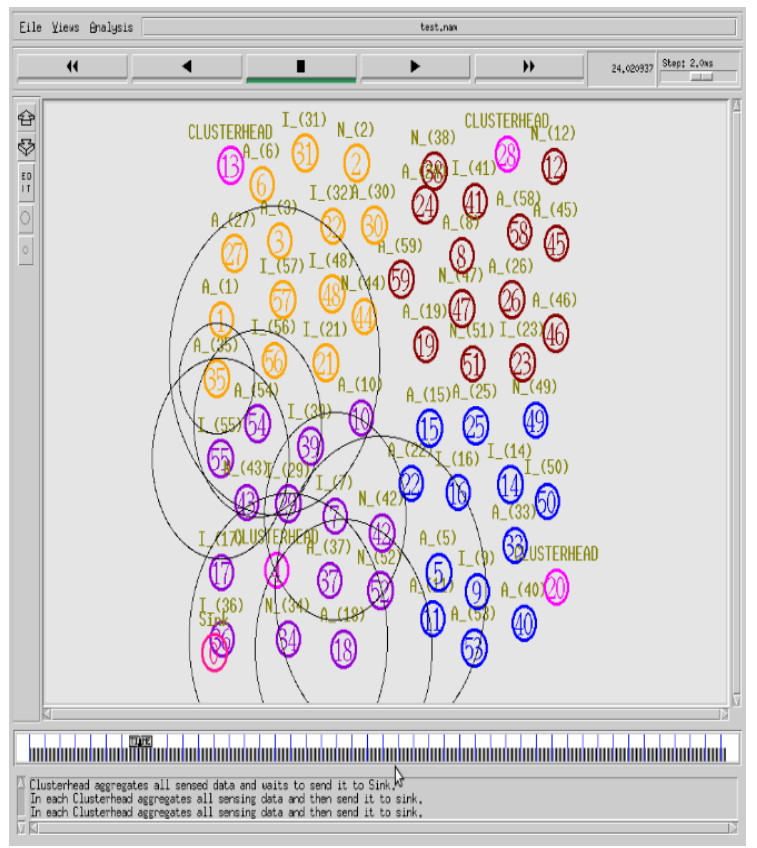

Figure 6: Data Transfer without Sink MobilityWith Sink Mobility

Sink moves to collect data from cluster head namely Cluster Head 4. 


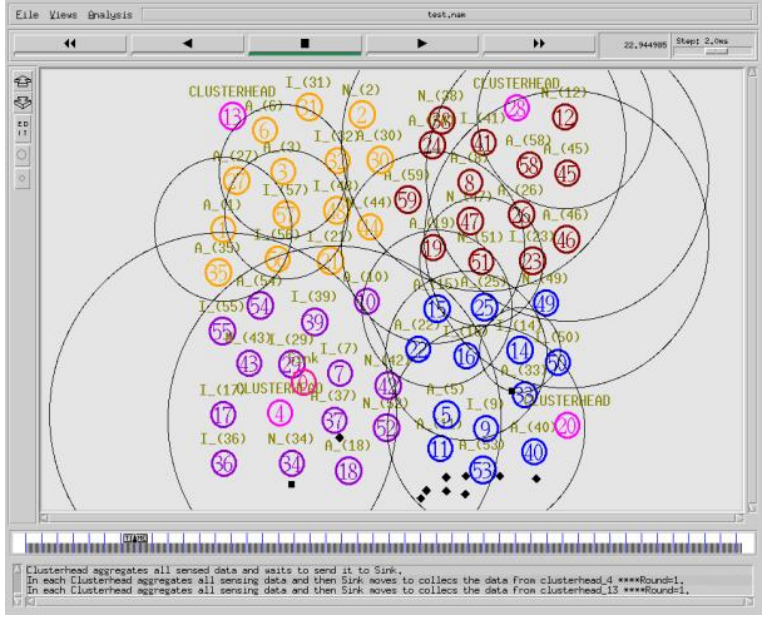

Figure 7: Data Transfer with Sink Mobility

\subsection{Comparative Graph}

\section{Network Lifetime}

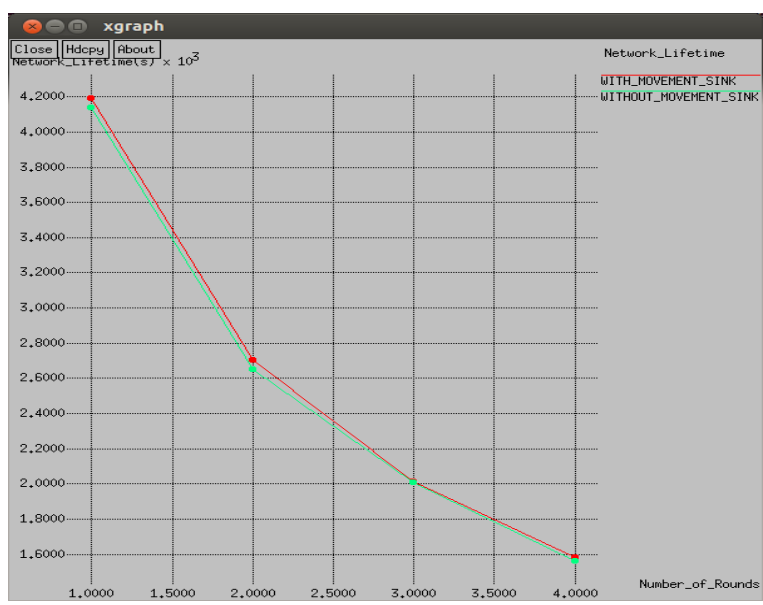

Figure 8: Network Lifetime with and without Sink Mobility

When the number of rounds increased the network lifetime is decreased. The proposed approach with mobility scheme provides better network lifetime when compared to the existing without mobility scheme.

\section{Stability Period}

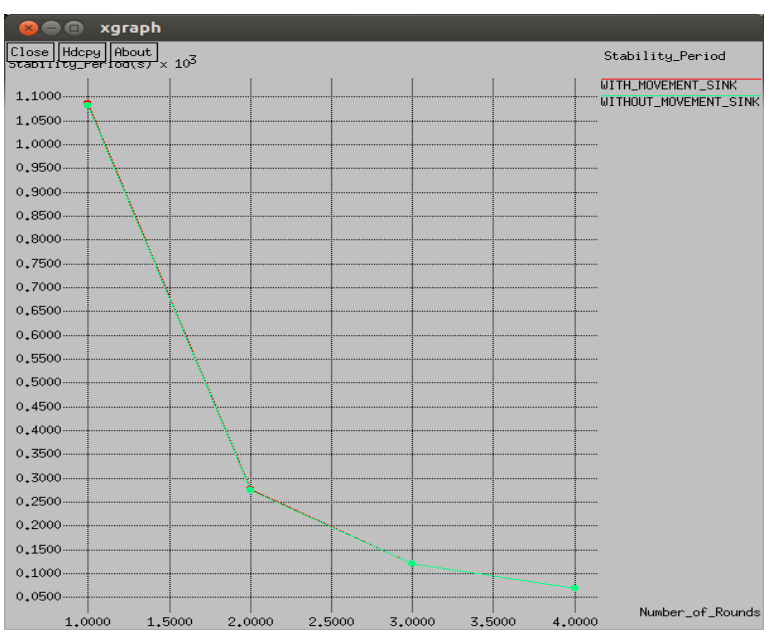

Figure 9: Stability Period with and without Sink Mobility
When the number of rounds increased the stability period is decreased. The proposed approach with mobility scheme and existing without mobility scheme provides similar performance.

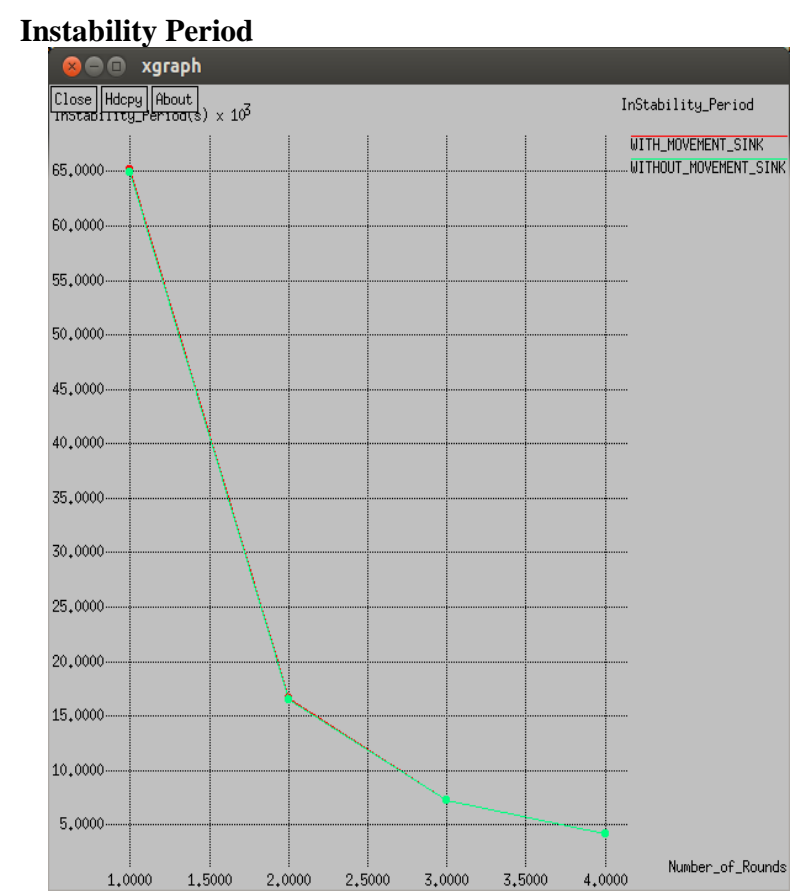

Figure 10: Instability Period with and without Sink Mobility

When the number of rounds increased the instability period is decreased. The proposed approach with mobility scheme and existing without mobility scheme provides similar performance.

\section{Throughput}

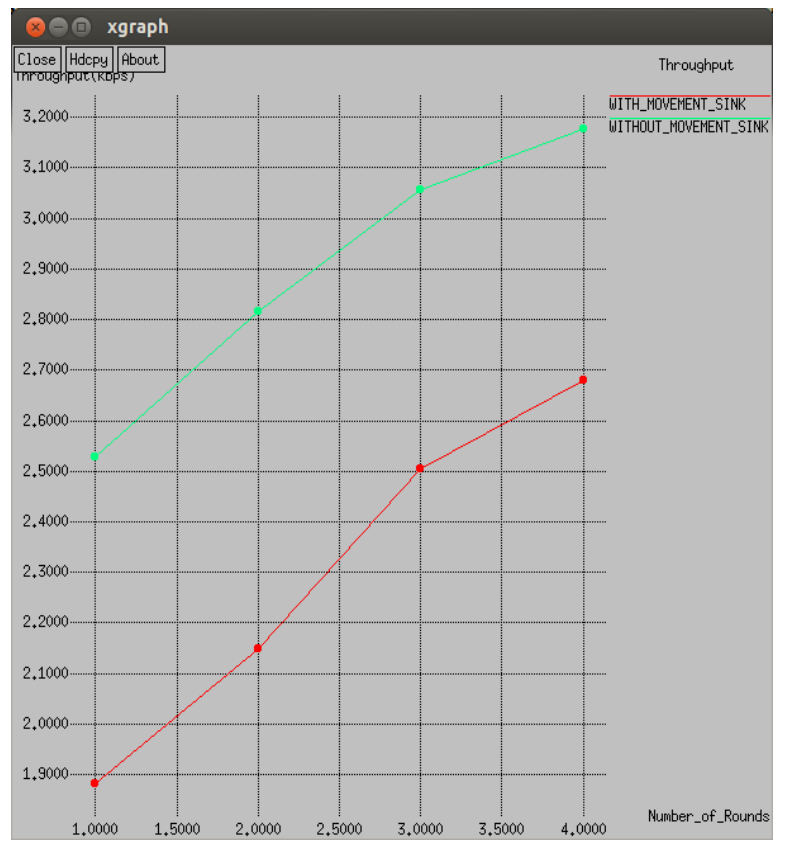

Figure 11:Throughput of Network with and without Sink Mobility

When the number of rounds increased the throughput is increased. The proposed approach with mobility scheme 
provides improved throughput when compared to the existing without mobility scheme.

\section{CONCLUSION}

ETSSEP, a reactive routing protocol is proposed where nodes considered with three different levels of energy. It is based on dynamic changing of cluster head election probability. It selects cluster heads on the basis of residual energy level of nodes and minimum number of clusters per round. The multiple cluster association technique is contributed to proposed approach in ETSSE protocol which is called degree of dependency based data transmission. Mobile Sink is being used for accumulating data from the Cluster Heads to be delivered to Base Station. It leads to increase in throughput and network life. We also evaluate and compare its performance with static sink WSN and WSN with random sink mobility. Our results show that the algorithm achieves better WSN lifetime compared to static sink case and random movement strategy. The usage of mobile sink in the WSN reduces the energy consumption of the nodes and to prevent the formation of wireless sensor networks. In comparison with existing protocol it can be concluded that this protocol will perform well in small as well as large sized networks.

\section{FUTURE WORKS}

Our proposed approach using ETSSEP gives better and improved performance as compared to ETSSEP. If we adjust the mobility of sink according to the cluster head location and by increasing the level of nodes classification in term of energy level we would be able to improve the stability and life time of the network. This makes that in future, we would like to modify proposed scheme to handle some other issues such as handling different Weighted Rendezvous Points for Data Collection.

\section{ACKNOWLEDGEMENT}

The euphoria of successfully completing this research work would be incomplete without expressing our gratitude to those who have helped us to achieve this milestone. We take this opportunity to express our deepest gratitude and appreciation to all those who helped us directly or indirectly towards the successful completion of this research work.

\section{REFERENCES}

[1] Awwad, S. A., Ng, C. K., Noordin, N. K., \& Rasid, M. F. A. (2011). Cluster based routing protocol for mobile nodes in wireless sensor networks. Wireless Personal Communications, 61(2), 251-281.

[2] Al-Karaki, J. N., \& Kamal, A. E.(2004). Routing techniques in wireless sensor networks: A survey. IEEE Wireless Communications, 11(6), 6-28.

[3] Afsar, M. M., Mohammad, H., \& Tayarani, N. (2014). Clustering in sensor networks: A literature survey. Journal of Network and Computer Applications, 46, 198226

[4] Bandyopadhyay, S., \& Coyle, E. J. (2003). An energy efficient hierarchical clustering algorithm for wireless sensor networks. In Proceedings of INFOCOM.

[5] Kashaf, A., Javaid, N., Khan, Z. A., \& Khan, I. A. (2012). TSEP: Threshold-sensitive stable election protocol for WSNs. In 10th international conference on frontiers of information technology (FIT) (Vol.164, no. 168, pp. 17-19)
[6] Femi, A. A., \& Jeremiah, D. D. (2011). An enhanced Stable Election Protocol (SEP) for clusteredheterogeneous WSN. Department of Information Science, University of Otago, New Zealand.

[7] Heinzelman, W. R., Chandrakasan, A., \& Balakrishnan, H. (2000). Energy-efficient communication protocol for wireless micro sensor networks. System Sciences. In Proceedings of the $33^{\text {rd }}$ annual Hawaii International conference (pp. 4-7).

[8] Islam, M. M., Matin, M. A., \& Mondol, T. K. (2012). Extended Stable Election Protocol (SEP) for three-level hierarchical clustered heterogeneous WSN.In IET conference on wireless sensor systems (WSS 2012) (pp. $1-4)$.

[9] Bandyopadhyay, S., \& Coyle, E. J. (2004). Minimizing communication costs in hierarchically-clustered networks of wireless sensors. Computer Networks, 44, 1-16.

[10] Kumar, D., Aseri, T. C., \& Patel, R. B. (2009). EEHC: Energy efficient heterogeneous clustered scheme for wireless sensor networks. Computer Communications, 32(4), 662-667.

[11] Kuila, P., \& Jana, P. K. (2014). A novel differential evolution based clustering algorithm for wireless sensor networks. Applied Soft Computing, 25, 414-425.

[12] Manjeshwar, A., \& Agarwal, D. P. (2001). TEEN: A routing protocol for enhanced efficiency inwireless sensor networks. In 1st international workshop on parallel and distributed computing issues in wireless networks and mobile computing.

[13] Qureshi, T. N., Javaid, N., Malik, M., Qasim, U., \& Khan, Z. A. (2012). On performance evaluation of variants of DEEC in WSNs. In Seventh international conference on broadband, wireless computing, communication and applications (BWCCA) (Vol. 12, no. 14, pp. 162-169)

[14] Saini, P., \& Sharma, A. K. (2010). Energy efficient scheme for clustering protocol prolonging the lifetime of heterogeneous wireless sensor networks. International Journal of Computer Applications, 6(2), 30-36.

[15] Sajjanhar, U., \& Mitra, P. (2007). Distributive energy efficient adaptive clustering protocol for wireless sensor networks. In Proceedings of the 2007 international conference on mobile data management (pp.26-33).

[16] Smaragdakis, G., Matta, I., \& Bestavros, A. (2004). SEP: A stable election protocol for clustered heterogeneous wireless sensor networks. In Second international workshop on sensor and actor network protocols and applications (SANPA 2004), Boston, MA.

[17] Shepard, T. J. (1996). A channel access scheme for large dense packet radio networks. In Proceedings of ACM SIGCOMM (pp. 219-230).

[18] P.Kanagaraj, T.Sasi. Energy Efficient Multiple Mobile Sink and Interference Aware Path Selection in Wireless Sensor Networks. IEEE Sponsored International Conference on Intelligent Systems and Control (ISCO'15).

[19] L.Brindha, U.Muthaiah. Energy Efficient Mobile Sink Path SelectionUsing a Cluster Based Approach in WSNs. 
International Journal of Innovative Research in Computer and Communication Engineering.

[20] Hamidreza Salarian, Kwan-Wu Chin, and Fazel Naghdy. An Energy-Efficient Mobile-Sink Path Selection Strategy for Wireless Sensor Networks. IEEE Transactions on Vehicular Technology, VOL. 63, NO. 5, JUNE 2014.

[21] Amruta S. Pattanshetti, Mr. N D. Kale. A Survey on BigData Gathering using Mobile Collector in Densely Deployed Wireless Sensor Network .International Journal of Engineering Research \& Technology (IJERT),Vol. 3 Issue 12, December-2014.
[22] Suchita R.Wankhade and Nekita A.Chavha. A Review on Data Collection Method with Sink Node in Wireless Sensor Network. International Journal of Distributed and Parallel Systems (IJDPS) Vol.4, No.1, January 2013.

[23] G. Sunil, Mrs. D. Jasmine David. Cluster Based Energy Efficient Sensory Data Collection With Mobile Sink International Journal of Advanced Research in Computer Engineering \& Technology (IJARCET) Volume 2, Issue 2, February 2013 